\title{
The intra-neuroendoscopic technique (INET): a modified minimally invasive technique for evacuation of brain parenchyma hematomas
}

Yujuan Zhang ${ }^{1}$, Ai-Jun Shan ${ }^{2}$, Yu-Ping Peng ${ }^{3}$, Pengfei Lei ${ }^{2}$, Jianzhong $\mathrm{Xu}^{2}$, Xianliang Zhong ${ }^{2}$ and Bo Du ${ }^{2 *}$ (1)

\begin{abstract}
Background: Minimally invasive endoscopic hematoma evacuation is widely used in the treatment of intracerebral hemorrhage. However, this technique still has room for improvement. The intra-neuroendoscopic technique (INET) is a modified minimally invasive technique, and we report its safety and efficacy in evacuating brain parenchyma hematomas by comparing it with cranial puncture and drainage operation (CPDO).

Methods: The frontal, temporal, or occipital approaches were used according to the site of bleeding. The preoperative and postoperative hematoma volumes, Glasgow Coma Scale (GCS) score, Cerebral State Index (CSI), hematoma evacuation rate, operation time, complications, and 30-day mortality and Glasgow Outcome Scale (GOS) were retrospectively compared between the two groups.

Results: A total of 98 patients were enrolled. The evacuation rate ( $84 \pm 7.1 \%$ versus $51.0 \pm 8.4 \%, p=0.00)$, 7 -day GCS $(11.8 \pm 1.2$ versus $10.4 \pm 1.5, p=0.01)$, and CSI ( $87.1 \pm 8.7$ versus $80.6 \pm 10.2, p=0.02)$ were higher, and the 30 -day mortality rate $(1.9 \%$ versus $15.6 \%, p=0.036)$ was lower in the INET group. However, the operation time was longer in the INET group than in the control group ( $65.2 \pm 12.5$ min versus $45.6 \pm 10.9 \mathrm{~min}, p=0.000)$. Multivariable logistic regression showed that a good medium-term outcome (GOS scores 4-5) was significantly associated with INET (odds ratio (OR) 3.514, 95\% confidence interval $(\mathrm{Cl}) 1.463-8.440, p=0.005$ ), age under 65 years (OR 1.402, 95\% Cl, 1.041-1.888, $p=0.026)$, and hematoma volume less than $50 \mathrm{ml}(\mathrm{OR} 1.974,95 \% \mathrm{Cl} 1.302-2.993, p=0.001)$.
\end{abstract}

Conclusions: INET surgery for brain parenchyma hematoma evacuation is a safe and efficient modified technique. This technique is minimally invasive, has less complications, and may be helpful in providing optimal outcomes for selected patients.

Trial registration: ClinicalTrials.gov, NCT02515903. Registered on 5 August 2015.

Keywords: Intra-neuroendoscopy technique (INET), Transparent sheath, Brain parenchyma hematoma, Minimally invasive surgery, Outcome

\footnotetext{
* Correspondence: ftipa@szhospital.com

${ }^{2}$ Department of Emergency, Shenzhen People's Hospital, The Second Clinical Medical College of Jinan University, The First Affiliated Hospital of Southern University of Science and Technology, Shenzhen 518020, Guangdong, China Full list of author information is available at the end of the article
}

(c) The Author(s). 2019 Open Access This article is distributed under the terms of the Creative Commons Attribution 4.0 International License (http://creativecommons.org/licenses/by/4.0/), which permits unrestricted use, distribution, and reproduction in any medium, provided you give appropriate credit to the original author(s) and the source, provide a link to the Creative Commons license, and indicate if changes were made. The Creative Commons Public Domain Dedication waiver (http://creativecommons.org/publicdomain/zero/1.0/) applies to the data made available in this article, unless otherwise stated. 


\section{Introduction}

Spontaneous intracerebral hemorrhage (ICH) is one of the leading causes of mortality and morbidity [1], with approximately $40 \% 1$-month mortality rates [2-4]. Furthermore, over $60 \%$ of surviving patients are reported to have moderate to severe disability [5]. Hypertensive hemorrhages are the most important risk factor for $\mathrm{ICH}$, as well as other causes of bleeding, including cerebral vascular malformations and cerebral aneurysms. The site of brain parenchyma hemorrhage is mainly located in the basal ganglia area (including the putamen and thalamus), frontal lobe, and occipital lobe. ICH requires a high level of medical care, making it one of the most costly neurological diagnoses [6]. Given the requirement for better treatment options for this common and devastating condition, treatment strategies and methods remain controversial despite extensive research on ICH treatment.

Theoretically, surgical evacuation of hematomas could reduce many secondary injuries associated with $\mathrm{ICH}$, such as those caused by its mass effects and the hemotoxicity of the blood breakdown products. However, the traditional craniotomy hematoma evacuation surgery did not show superiority in some randomized trials [7]. The long surgical duration and great damage to viable tissue in traditional craniotomy hematoma evacuation may offset the beneficial effects of the procedure, especially in the case of a deep-seated brain parenchyma hematoma $[8,9]$. Therefore, minimally invasive surgical techniques that could shorten the surgical duration are more acceptable and are being investigated. Recently, the following two minimally invasive approaches have mainly been used: the cranial puncture and drainage operation (CPDO) and the endoscopic hematoma evacuation operation (EHEO). The CPDO was demonstrated to have beneficial effects for spontaneous intracerebral hemorrhage patients in a small randomized controlled trial and a meta-analysis [10, 11]. In recent years, with improvements in endoscopic technology and equipment, the EHEO has been regarded as a more promising method to treat ICH [12-18]. Compared with the CPDO, the endoscopic approach can achieve immediate hematoma evacuation, reducing the possibility of cumulative secondary injuries $[9,19]$. In addition, the endoscopic approach has an impressive short-term outcome, low re-hemorrhaging rate, and less surgery-related morbidity [12, 13, 15, 16, 18, 20-22].

Reviewing these reported case series, we noted the following. (1) The sheath is different. Some groups used a metal sheath, whereas others used a transparent sheath. The latter is superior to the former in many ways. However, its diameter is approximately $20 \mathrm{~mm}$, and the surgery requires the removal of a 3-cm diameter bone flap, necessitating allogeneic material fixation after the operation [23]. (2) During the process of implanting the transparent sheath, most of the hematoma is not visible. (3) Endoscopic surgery is mainly carried out in an air environment, and an operation environment with continuous fluid flush is not easy to achieve. The surgical operation is performed in the transparent sheath but outside of the endoscope, whose only role is to provide light. (4) It is still challenging to remove hard hematomas.

In our hospital, we started performing endoscopic hematoma evacuation surgery in September 2015. We have developed a transparent sheath (Chinese Patent No. ZL 200820046232.0, State Intellectual Property Office of P.R. China, website http://cpquery.sipo.gov.cn) for largechannel neuroendoscope, which achieved a visible puncture. We also developed a hematoma smashing suction apparatus (Chinese Patent No. ZL 201520248717.8, State Intellectual Property Office of P.R. China, website http:// cpquery.sipo.gov.cn) to solve the problem of removing hard hematomas. We designed a non-randomized concurrent control trial, where the control was a CPDO group. The clinical efficacy and safety of the new method were discussed based on this study.

\section{Material and methods \\ Ethics statement}

The Medical Science Ethics Committee of the Southern Medical University approved this study (NFEC-2015-034). Each patient or an appropriate family member provided informed written consent to obtain clinical materials.

\section{Study population}

From September 2015 to May 2018, a total of 98 patients met the inclusion criteria for the study, with 53 patients in the intra-neuroendoscopic technique (INET) treatment group and 45 patients in the control group. All patients were enrolled from the neurosurgery department of Nanfang Hospital and the emergency center of Shenzhen People's Hospital. The baseline data of the two groups are shown in Table 1. All patients underwent brain computed tomography $(\mathrm{CT})$ to determine the diagnosis, and magnetic resonance angiography (MRA), CT angiography, or digital subtraction angiography (DSA) was performed to rule out patients with an arteriovenous malformation or an aneurysm. The Tada formula [24] was used to calculate the hematoma volume.

\section{Inclusion and exclusion criteria Inclusion criteria}

The inclusion criteria are as follows: (1) Diagnosis of spontaneous hemorrhage in the parenchyma of the brain on CT scan. (2) Hemorrhage volume was greater than $25 \mathrm{ml}$, and no brain hernia was formed. (3) Age range was 40-75 years. (4) Hemorrhagic duration (from stroke onset to hospital) was less than $72 \mathrm{~h}$. (5) Informed consent was provided from patients and/or their relatives. 
Table 1 Comparison of baseline indicators between the INET and control groups

\begin{tabular}{llll}
\hline Baseline indicators & $\begin{array}{l}\text { INET group } \\
(n=53)\end{array}$ & $\begin{array}{l}\text { Control group } \\
(n=45)\end{array}$ & $p$ value \\
\hline Age $\leq 65$ years & $27(50.9 \%)$ & $33(73.3 \%)$ & 0.023 \\
Preoperative GCS & $8.9 \pm 1.4$ & $8.3 \pm 1.6$ & 0.432 \\
Preoperative hematoma volumes $\leq 50 \mathrm{ml}$ & $40(75.5 \%)$ & $71.9 \pm 7.4$ & 0.038 \\
Preoperative CSI & $76.1 \pm 7.9$ & & 0.337 \\
Hemorrhage sites & & $33(73.3 \%)$ & 0.809 \\
Basal ganglia & $40(75.4 \%)$ & $4(8.9 \%)$ & 0.951 \\
Frontal lobe & $6(11.3 \%)$ & $3(6.7 \%)$ & 0.829 \\
Parietal lobe & $3(5.7 \%)$ & $5(11.1 \%)$ & 0.797 \\
Occipital lobe & $4(7.5 \%)$ & \\
\hline
\end{tabular}

\section{Exclusion criteria}

The exclusion criteria are as follows: (1) Disturbance in blood coagulation, such as thrombocytopenia or hepatitis; (2) intracranial or general infection; (3) co-existence of severe heart, liver, kidney, or lung disease or functional failure; (4) a previous history of stroke with neurological deficits; (5) under anticoagulation therapy; and (6) hemorrhage caused by brain injury, intracranial aneurism or cerebral arteriovenous malformations.

\section{Surgical techniques}

The INET equipment used in this study consisted of a high-definition imaging system, cold light source, Zeppelin large-working-channel endoscope, endoscope-dedicated bipolar coagulator (ZNE-242BIP, Zeppelin, Germany), and two of our patented inventions, namely, a transparent sheath (Chinese Patent No. ZL 200820046232.0) and a hematoma smashing aspirator (Chinese Patent No. ZL 201520248717.8). The Zeppelin large-working-channel endoscope (model number NEH/30-177-6.5) has a $177-\mathrm{mm}$ working length, $6.5-\mathrm{mm}$ body diameter, and $0^{\circ}$ or $30^{\circ}$ angle of view. The endoscope's working channel diameter is $3.7 \mathrm{~mm}$, and two $1.5-\mathrm{mm}$ suction/flushing channels are integrated into the endoscope. The transparent sheath can be seamlessly fit with the neuroendoscope, its outer diameter is $7 \mathrm{~mm}$, and it has an adjustable length. A transparent tip and a fixed device were also designed for the sheath. After a successful endoscope-guided puncture, the tip of the sheath can be removed together with the endoscope (Fig. 1). The diameter of the hematoma smashing aspirator, which contains a spiral suction device, is $3.0 \mathrm{~mm}$, and it can be connected to a power system (Fig. 2).

The INET operation method for hematoma evacuation was performed as follows: the puncture point was selected
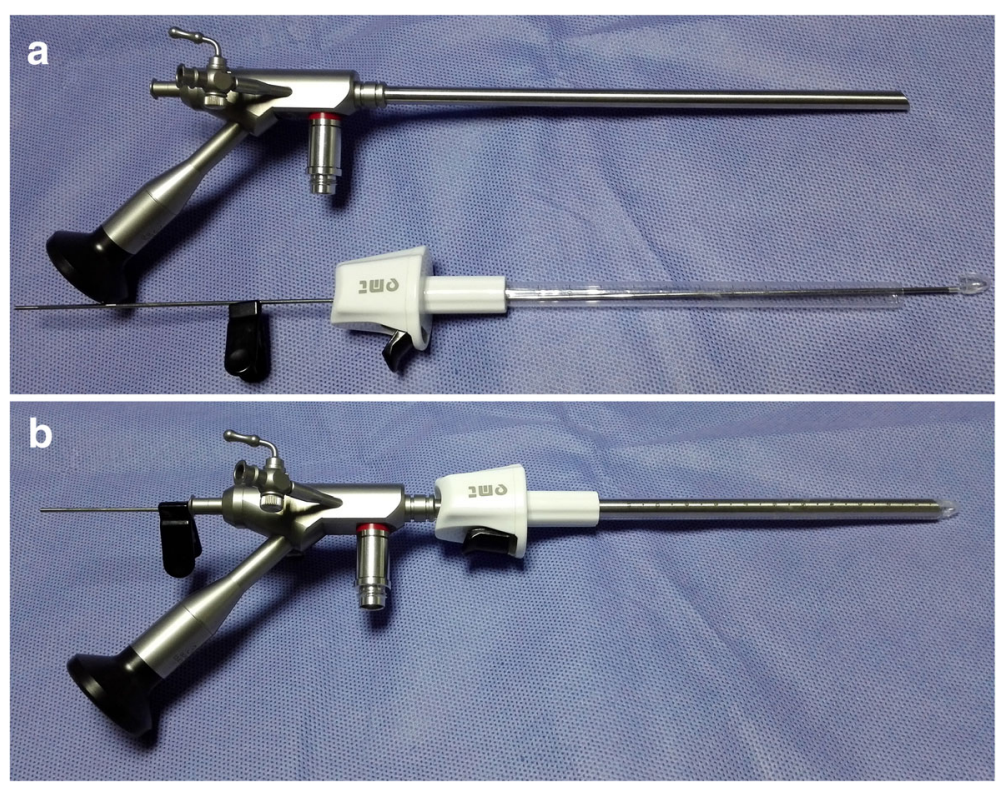

Fig. 1 Transparent sheath and neuroendoscope before (a) and after (b) assembly 


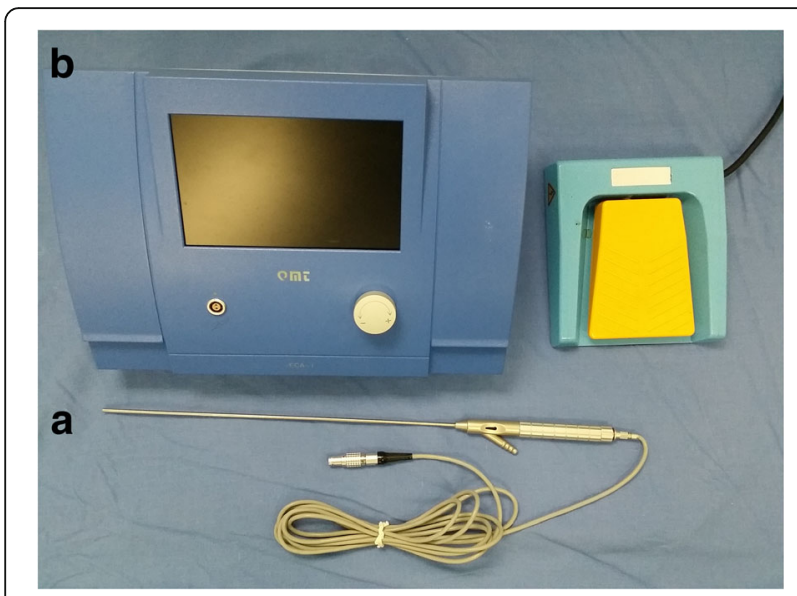

Fig. 2 Hematoma smashing aspirator. a Smashing device (3.0-mm diameter). $\mathbf{b}$ Power system. The suction can be controlled by both a gear and a pedal

according to the cerebral CT scan and was usually at the site where the hematomas had the shortest distance to the skin. Typically, the site was not close to large blood vessels and functional areas. We performed a $2.5-3 \mathrm{~cm}$ straight scalp incision and drilled a 1-cm bone hole, and the puncture process was performed visually (Fig. 3). During the puncture, the blood vessels in front could be avoided, and the margin and cavity of the hematoma were clearly visible (Fig. 4). After puncture, we removed the neuroendoscope together with the transparent sheath tip and placed the neuroendoscope again to evacuate the hematomas through the working channel. First, the most fragile hematomas were sucked and evacuated with the normal suction tube, and the hard hematomas were carefully cleared with the hematoma smashing suction apparatus. The suction force of the smashing suction apparatus could be manually controlled, and its inner axis could be adjusted to a shorter length than the sheath to help avoid vessel injury caused by improper suction. The small amount of bleeding stopped after the hematoma cavity was continuously washed with $39^{\circ} \mathrm{C}$ saline solution. The active bleeding was stopped with an endoscopy-specific bipolar coagulation device. The clot was carefully removed under alternating conditions of air and water until the surrounding brain tissues were exposed. When the hematomas were removed, the brain tissue of the hematoma cavity wall could be seen through the transparent sheath (Additional file 1: Video 1). Finally, we carefully withdrew the sheath and routinely indwelled a drainage tube.

The CPDO method for hematoma evacuation was performed as follows: a set of hard channel puncture instruments (Wantfu Co. Ltd, Beijing, China, standard number YZB/State 1699-2014) was used to remove hematomas. The puncture needle consists of a double channel enclosed in a cannula with small holes at the end to allow infusion of lysis fluid (Fig. 5). The puncture site was based on CT scans of the patient and aimed to avoid functional domains and blood vessels. The puncture needle was fixed onto the skull after the needle point had been located in the center of the hematoma. As much of the hematoma as possible was aspirated. Lysis fluid was then injected under pressure to dissolve the residual hematoma to enable easy aspiration through the needle. The main component of the lysis fluid was urokinase $(10,000-50,000 \mathrm{U}$ based on the volume of hemorrhage) [25]. CT scanning was performed less than $12 \mathrm{~h}$ after the procedure to calculate the hematoma evacuation rate and again 1-3 days later to ascertain the position of the puncture needle and estimate the volume
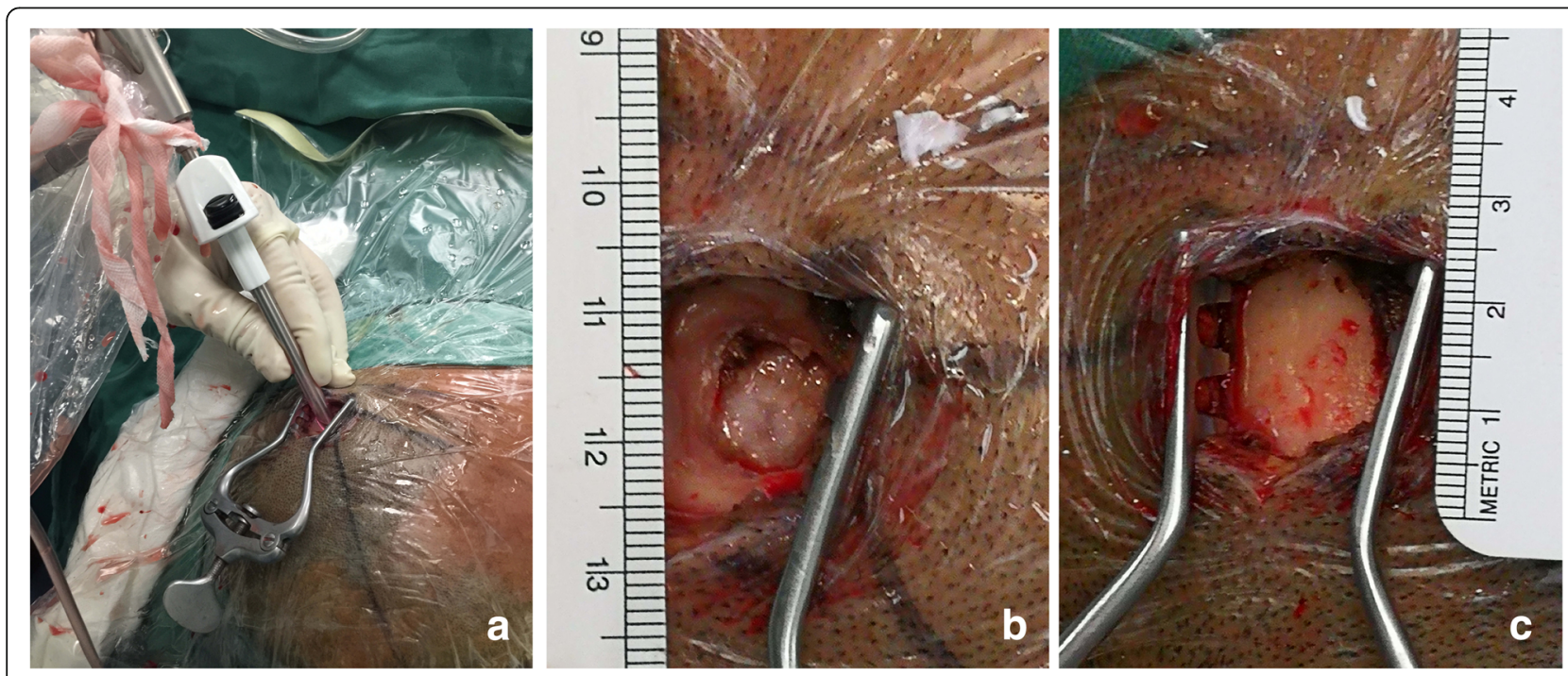

Fig. 3 a Scalp incision $(2.5-3.0 \mathrm{~cm})$. b Bone hole $(1 \mathrm{~cm})$. c Endoscopic guided puncture 

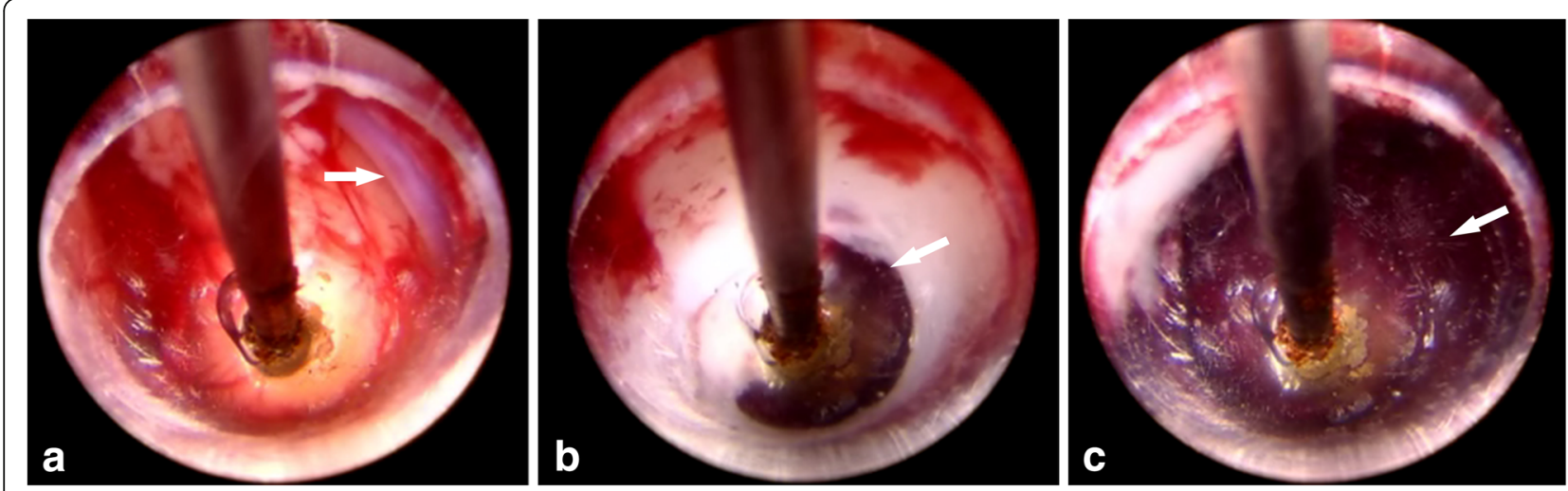

Fig. 4 Visible hematoma puncture with INET. a Effective avoidance of a vessel (arrow) in front during puncture. $\mathbf{b}$ The endoscope carefully entered the edge of the brain parenchyma hematoma (arrow). c The endoscope completely entered the center of hematoma cavity (arrow)

of any remaining blood. The drainage needle was retained in the brain for 3-5 days.

There was no difference in medical treatment between the two study groups. Hypertension was controlled early in the course of therapy if the systolic blood pressure was greater than $140 \mathrm{mmHg}[26,27]$.

\section{Outcome data}

After surgery, the patients were sent to the intensive care unit (ICU) for medical treatment. CT scans were obtained less than $12 \mathrm{~h}$ postoperatively. The preoperative and postoperative hematoma volumes, hematoma evacuation rate, Glasgow Coma Scale (GCS) scores, Cerebral State Index (CSI), and complications (gastrointestinal stress ulcer bleeding, intracranial gas accumulation, intracranial infection, and cerebrospinal fluid leakage) were recorded 7 days after the operation. The follow-up surveys were performed 30 days after surgery. The 30-day mortality and Glasgow Outcome Scale (GOS) scores were collected from the patients' medical records upon readmission, from the outpatient clinic records, or via telephone interview. GOS scores of 4-5 were defined as a good outcome, and GOS scores of 1-3 were defined as a bad outcome in this study.

\section{Statistics}

The preoperative and postoperative hematoma volumes, operation time, preoperative GCS/CSI scores, and postoperative 7-day GCS/CSI scores were compared using an independent-sample $t$ test. The 30-day mortality, hematoma evacuation rate, and complications were analyzed using the chi-squared test and Fisher's exact test. Multivariable logistic regression model was constructed to analyze the association between the 30-day GOS and INET, age, and preoperative hematoma volumes. The data are reported as odds ratios (ORs) and 95\% confidence intervals (CIs). Statistical analyses were performed using SPSS 13.0 software
(SPSS Inc., Chicago, IL, USA). The threshold for statistical significance was set at $p=0.05$.

\section{Results \\ Clinical manifestations}

A total of 98 patients were enrolled in this study, and the baseline indicators were compared between the two groups (Table 1). The proportions of patients under 65 years $(p=0.023)$ and with preoperative hematoma volumes less than $50 \mathrm{ml}(p=0.038)$ were significantly different between the two groups. The preoperative GCS scores and CSI showed no significant difference between the two groups. The patient bleeding sites or the major body of the hematoma were mainly located in the basal ganglia $(75.4 \%$ in the INET group and $73.3 \%$ in the control group), and a few were distributed in the frontal lobe, temporal lobe, parietal lobe, and occipital lobe. There was no significant difference in the distribution of bleeding sites between the two groups.

\section{Short-term outcomes}

The scalp incision and bone hole diameter were slightly larger in the INET group (Table 2). The operation time in the INET group was longer than that in the control group $(65.2 \pm 12.5 \mathrm{~min}$ versus $45.6 \pm 10.9 \mathrm{~min}, p=0.00)$, but the hematoma clearance rate was significantly higher $(84 \pm 7.1 \%$ versus $51 \pm 8.4 \%, p=0.00)$. The 7 -day GCS scores $(12.1 \pm 1.65$ versus $10.8 \pm 1.5, p=0.01)$ and CSI $(88.7 \pm 5.9$ versus $80.1 \pm 6.3, p=0.02)$ were higher in the INET group.

\section{Medium-term outcomes and influencing factors}

The 30 -day mortality was lower (1.9\% versus $15.6 \%, p=$ $0.036)$ in the INET group than in the control group. The postoperative complication rates of gastrointestinal stress ulcer bleeding (15.1\% versus $24.4 \%, p=0.243)$ and cerebrospinal fluid leakage $(7.5 \%$ versus $4.4 \%, p=0.829)$ showed no significant difference between the two 


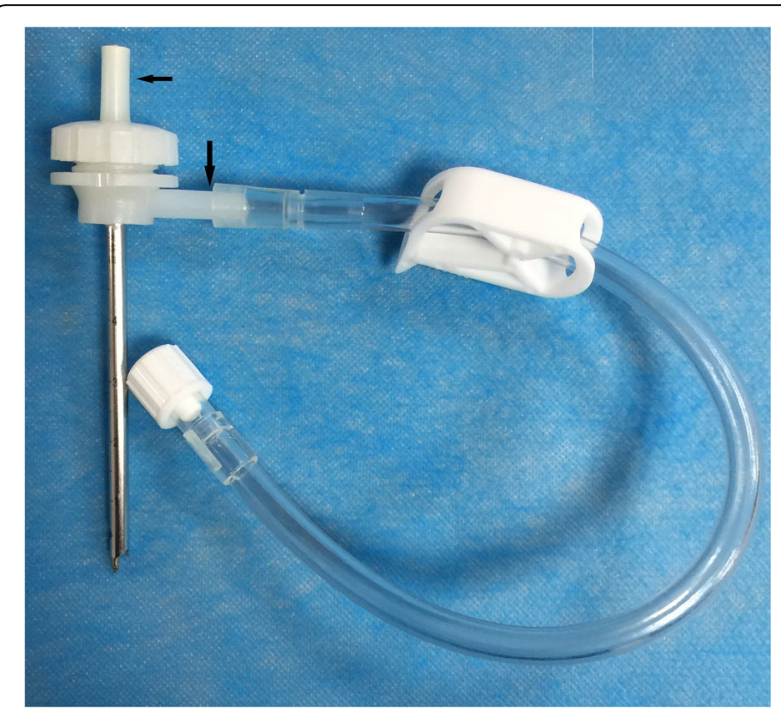

Fig. 5 Puncture needle was connected to a drainage tube. Arrow denotes double channels

groups. The incidence of intracranial gas accumulation after surgery was higher $(77.4 \%$ versus $11.1 \%, p=0.000)$, and the intracranial infection rate was lower $(3.8 \%$ versus $20.0 \%, p=0.011$ ) in the INET group than in the control group (Table 3 ).

Multivariable logistic regression analysis showed that a good medium-term outcome (GOS scores 4-5) was significantly associated with INET (OR 3.514, 95\% CI $1.463-8.440, p=0.005$ ), age under 65 years (OR 1.402, 95\% CI, 1.041-1.888, $p=0.026$ ), and hematoma volume less than $50 \mathrm{ml}$ (OR 1.974, 95\% CI 1.302-2.993, $p=$ 0.001) (Table 4).

\section{Discussion}

Neuroendoscopic techniques have progressed rapidly. The indication for operation has developed from the previous applications for intraventricular lesions and cystic lesions to those of brain parenchyma lesions. The endoscopic hematoma evacuation technique has been under development for nearly 20 years [9]. Several important technical developments were reported by various

Table 2 Comparison of intraoperation, postoperation, and 7-day follow-up indicators between the two groups

\begin{tabular}{llll}
\hline Indicators & $\begin{array}{l}\text { INET group } \\
(n=53)\end{array}$ & $\begin{array}{l}\text { Control group } \\
(n=45)\end{array}$ & $p$ value \\
\hline Scalp incision length (cm) & $2.5-3.0$ & $0.5-1.0$ & N/A \\
Skull drilling diameter (cm) & $0.8-1.1$ & $0.2-0.3$ & N/A \\
Operation time (min) & $65.2 \pm 12.5$ & $45.6 \pm 10.9$ & 0.00 \\
Evacuation rate (\%) & $84 \pm 7.1 \%$ & $51 \pm 8.4 \%$ & 0.00 \\
7-day GCS & $12.1 \pm 1.6$ & $10.8 \pm 1.5$ & 0.01 \\
7-day CSI & $88.7 \pm 5.9$ & $80.1 \pm 6.3$ & 0.02 \\
\hline
\end{tabular}

groups to enhance their orientation, visualization, and safety $[9,22]$. One of the most important inventions was the transparent sheath that was used as a working channel for neuroendoscope and surgical instruments [14, 18]. In 2000, Nihishara first reported this improvement [18], and it was widely adopted by many surgeons for endoscopic hematoma evacuation surgery $[13,15,20$, $22,28-30]$. Compared with a steel sheath, the transparent sheath has the obvious advantage that the residual hematoma and hematoma-brain border can be easily identified through the transparent wall of the sheath (Additional file 1: Video 1). A deeper hematoma cavity can be clearly identified through the endoscope, which helps reduce bleeding and improve effectiveness and safety. This technique was first called neuroendoscopic control technology (NECT). In NECT, all the surgical instruments were used outside of the endoscope, and the main role of the endoscope was illumination. This surgical technique to remove the hematoma is effective, but several features, such as its non-visual implantation, larger sheath diameter, and difficulty in removing hard hematomas, still have room for improvement. In this study, we improved the transparent sheath and developed a hematoma smashing suction apparatus to achieve more minimally invasive removal of the hematoma.

\section{Summary of INET surgical capabilities}

From this study, we can summarize the capabilities of INET as follows: (1) The puncture location was visualized, and the hematomas were evacuated from their center, as the vessels responsible for bleeding were mostly located around the hematoma cavity margin. (2) The brain tissue was white, and the hematomas were dark brown under endoscope. Most hematomas could be evacuated quickly in the air condition. If the boundary between the hematomas and brain tissue was not clearly identified, the hematoma cavity was washed using $39^{\circ} \mathrm{C}$ saline and changed to the water condition to continue the evacuation. We found that real-time adjustment of the depth and angle of the transparent sheath in the hematoma cavity was secure. When we withdrew the sheath after the surgery, the bleeding sites in the puncture path were electrocoagulated. (3) After the operation, we routinely washed the hematoma cavity with 39 ${ }^{\circ} \mathrm{C}$ saline. Small amounts of bleeding were stopped with continuous washing. The active bleeding was stopped with endoscopy bipolar coagulation. (4) Removing the hematomas too quickly or forcefully evacuating clots adhering to vessels might lead to uncontrolled bleeding.

\section{Cerebral State Index (CSI)}

CSI was obtained with a UP-8000 cerebral state monitor (the handset was provided by Danmeter AS Ltd, Denmark, and the mainframe was provided by the anesthesia depth 
Table 3 Comparison of postoperative complication rates and mortality during 30 days in the two groups

\begin{tabular}{llll}
\hline Postoperative complication & $\begin{array}{l}\text { INET group } \\
(n=53)\end{array}$ & $\begin{array}{l}\text { Control group } \\
(n=45)\end{array}$ & $p$ value \\
\hline Gastrointestinal stress ulcer bleeding & $8(15.1 \%)$ & $11(24.4 \%)$ & 0.243 \\
Intracranial gas accumulation & $41(77.4 \%)$ & $5(11.1 \%)$ & 0.000 \\
Intracranial infection & $2(3.8 \%)$ & $9(20.0 \%)$ & 0.011 \\
Cerebrospinal fluid leakage & $4(7.5 \%)$ & $2(4.4 \%)$ & 0.829 \\
30-day mortality & $1(1.9 \%)$ & $7(15.6 \%)$ & 0.036 \\
\hline
\end{tabular}

lab of Shen-zhen Creative Industry Co., Ltd. The company was listed and registered in the Shenzhen Science and Technology Bureau). The instrument recorded spontaneous EEG through electrodes connected to the patient's forehead, temple, and mastoid (behind ear) and quantified the patient's level of consciousness and brain function through a fuzzy logic state analyzer. Our previous study [31] showed that CSI (0-100) could reflect the real-time coma depth and the coma dynamic process accurately; zero represented brain death, and 100 represented normal brain function. This study found that the whole brain function state showed obvious improvement after 7 days in the INET group (88.7 \pm 5.9 versus $80.1 \pm 6.3, p=0.02)$.

\section{Comparison of the INET group and the control group for evacuation of brain parenchyma hematomas}

The INET and CPDO methods both involve minimally invasive drilling techniques. In the present study, we safely achieved an average evacuation rate of $84 \pm 7.1 \%$, and the 30 -day mortality rate was $1.9 \%$ using INET. The evacuation rate reported by other groups utilizing similar techniques (i.e., transparent sheath and endoscope) ranged from 80 to $99 \%[14,16,18,28,29]$, and the 30 -day mortality rate ranged from 0 to $7.1 \%[8,11-13$, 20]. However, the hematoma evacuation rate in the control group was only $51 \pm 8.4 \%$, and its 30-day mortality was $15.6 \%$. Because of the high hematoma clearance rates in the INET group, the hematoma mass effects and the hemotoxicity of the blood breakdown products were promptly relieved. Seven days after surgery, the average patient GCS scores and CSI were more improved than those of the control group.

Table 4 Effect of INET on brain parenchyma hematoma patients with good outcomes (GOS score of 4-5)

\begin{tabular}{llll}
\hline Variable & OR & $95 \% \mathrm{Cl}$ & $p$ value \\
\hline INET applied (no) & Reference & & \\
INET applied (yes) & 3.514 & $1.463-8.440$ & 0.005 \\
Age $\geq 65$ years & Reference & & \\
Age $<65$ years & 1.402 & $1.041-1.888$ & 0.026 \\
Hematoma volume $\geq 50 \mathrm{ml}$ & Reference & & \\
Hematoma volume $<50$ & 1.974 & $1.302-2.993$ & 0.001 \\
\hline
\end{tabular}

$O R$ odds ratio, $\mathrm{Cl}$ confidence interval. ${ }^{*} p$ value of the Wald test
The comparison of postoperative complications over 30 days in the two groups showed that the rate of intracranial gas accumulation was higher in the INET group $(77.4 \%$ versus $11.1 \%, p=0.000)$, and the gas accumulation occurred mainly in the forehead subdural and surgical area. Rinsing with saline after surgery reduced the surgical area gas accumulation. The forehead subdural gas accumulation occurred due to the volume contraction of brain tissue when using negative pressure to remove hematomas. One way to prevent air from entering was to use wet cotton sheets as padding around the bone hole. The incidence of intracranial infection in the control group was significantly higher than that in the INET group (3.8\% versus $20.0 \%, p=0.011$ ), which was mainly due to the prolonged placement of the drainage tube and repeated injection of urokinase. The relatively longer operative time $(65.2 \pm 12.5 \mathrm{~min}$ versus $45.6 \pm 10.9 \mathrm{~min}, p=0.00)$ in the INET group than in the control group was not associated with an increased risk of cerebrospinal fluid leak $(7.5 \%$ versus $4.4 \%, p=0.829$ ).

Multivariable logistic regression analysis showed that INET (OR 3.514, 95\% CI 1.463-8.440, $p=0.005$ ), age under 65 years (OR 1.402, 95\% CI, 1.041-1.888, $p=0.026$ ), and hematoma volume less than $50 \mathrm{ml}$ (OR 1.974, 95\% CI 1.302-2.993, $p=0.001$ ) were independent predictors of good outcomes (GOS 4-5 scores) (Table 4). Therefore, INET surgery can provide a better outcome for patients with cerebral hemorrhage within 30 days, but determining the long-term prognosis of patients requires longer observations. Recently, Ma et al [23] used NECT to treat deep-seated basal ganglia hematomas and found that using their transparent sheath, which was different from ours, might have the potential to save patients and provide better long-term outcomes for patients with an ICH score of 3. Our transparent sheath was improved based on similar models; it was minimally invasive, and the hematoma removal efficiency of INET was similar.

\section{Limitations}

The present study does have some limitations. First, it was a non-randomized concurrent control study conducted in a single center. Second, although strict inclusion/exclusion criteria were set and statistical methods were used to control selection bias, we still could not avoid the occurrence 
of it. Third, the surgeons responsible for surgery are trained doctors, but differences in individual clinical skills are unavoidable. Therefore, the conclusions require confirmation in future multicenter randomized controlled trial.

\section{Conclusions}

For brain parenchyma hematomas, INET is a minimally invasive and safe surgical option. This modified technique with less complications might have the potential to save lives and provide better short- and medium-term outcomes for patients.

\section{Additional file}

Additional file 1: Video 1 . Brain tissue of the hematoma cavity wall is seen through the transparent sheath after removal of the hematoma. (WMV $1250 \mathrm{~kb}$ )

\section{Abbreviations}

$\mathrm{Cl}$ : Confidence interval; CPDO: Cranial puncture and drainage operation; CSI: Cerebral State Index; EHEO: Endoscopic hematoma evacuation operation; GCS: Glasgow Coma Scale; GOS: Glasgow Outcome Scale; ICH: Intracerebral hemorrhage; INET: Intra-neuroendoscopic technique; NECT: Neuroendoscopic control technology; OR: Odds ratio

\section{Acknowledgements}

This work was supported by the Department of Neurosurgery, Nanfang Hospital, Southern Medical University. The authors want to thank Prof. Songtao Qi for his technical support.

\section{Funding}

This study was funded by the Clinical Research Project of Shenzhen Health and Family Planning Commission in China (No. SZLY2018007), Shenzhen People's Hospital Young and Middle-aged Research Funds Cultivation Fund (No. SYKYPY201923), and Guangdong Medical Science and Technology Research Fund Project (No. A2016545).

\section{Availability of data and materials}

All the supporting data are available.

\section{Authors' contributions}

BD conceived and designed the whole experiments and manuscript. $Y Z$ and $\mathrm{BD}$ performed the major work and the assay. All authors read and approved the final manuscript.

\section{Ethics approval and consent to participate}

The Medical Science Ethics Committee of the Southern Medical University approved this study (NFEC-2015-034). Each patient or an appropriate family member provided informed written consent to obtain clinical materials.

\section{Consent for publication}

Yes

\section{Competing interests}

The authors declare that they have no competing interests.

\section{Publisher's Note}

Springer Nature remains neutral with regard to jurisdictional claims in published maps and institutional affiliations.

\section{Author details}

'Department of Ultrasound, Shenzhen People's Hospital, The Second Clinical Medical College of Jinan University, The First Affiliated Hospital of Southern University of Science and Technology, Shenzhen 518020, Guangdong, China. ${ }^{2}$ Department of Emergency, Shenzhen People's Hospital, The Second Clinical
Medical College of Jinan University, The First Affiliated Hospital of Southern University of Science and Technology, Shenzhen 518020, Guangdong, China. ${ }^{3}$ Department of Neurosurgery, Nanfang Hospital, Southern Medical University, Guangzhou, China.

Received: 14 February 2019 Accepted: 8 April 2019

Published online: 06 May 2019

\section{References}

1. Keep RF, Hua Y, Xi G. Intracerebral haemorrhage: mechanisms of injury and therapeutic targets. Lancet Neurol. 2012;11(8):720-31.

2. Broderick J, Connolly S, Feldmann E, Hanley D, Kase C, Krieger D, et al. Guidelines for the management of spontaneous intracerebral hemorrhage in adults: 2007 update: a guideline from the American Heart Association/ American Stroke Association Stroke Council, High Blood Pressure Research Council, and the Quality of Care and Outcomes in Research Interdisciplinary Working Group. Circulation. 2007;116(16):e391-413.

3. Sacco S, Marini C, Toni D, Olivieri L, Carolei A. Incidence and 10-year survival of intracerebral hemorrhage in a population-based registry. Stroke. 2009; 40(2):394-9.

4. van Asch CJ, Luitse MJ, Rinkel GJ, van der Tweel I, Algra A, Klijn CJ. Incidence, case fatality, and functional outcome of intracerebra haemorrhage over time, according to age, sex, and ethnic origin: a systematic review and meta-analysis. Lancet Neurol. 2010;9(2):167-76.

5. van Beijnum J, Lovelock CE, Cordonnier C, Rothwell PM, Klijn CJ, Al-Shahi Salman R. Outcome after spontaneous and arteriovenous malformationrelated intracerebral haemorrhage: population-based studies. Brain. 2009; 132(Pt 2):537-43.

6. Russell MW, Joshi AV, Neumann PJ, Boulanger L, Menzin J. Predictors of hospital length of stay and cost in patients with intracerebral hemorrhage. Neurology. 2006;67(7):1279-81.

7. Mendelow AD, Gregson BA, Rowan EN, Murray GD, Gholkar A, Mitchell PM. Early surgery versus initial conservative treatment in patients with spontaneous supratentorial lobar intracerebral haematomas (STICH II): a randomised trial. Lancet. 2013;382(9890):397-408.

8. Fiorella D, Zuckerman SL, Khan IS, Ganesh Kumar N, Mocco J. Intracerebral hemorrhage: a common and devastating disease in need of better treatment. World Neurosurg. 2015;84(4):1136-41.

9. Beynon C, Schiebel P, Bosel J, Unterberg AW, Orakcioglu B. Minimally invasive endoscopic surgery for treatment of spontaneous intracerebral haematomas. Neurosurg Rev. 2015;38(3):421-8 discussion 428.

10. Wang WZ, Jiang B, Liu HM, Li D, Lu CZ, Zhao YD, et al. Minimally invasive craniopuncture therapy vs. conservative treatment for spontaneous intracerebral hemorrhage: results from a randomized clinical trial in China. Int J Stroke. 2009:4(1):11-6.

11. Mould WA, JR Carhuapoma J, Muschelli KL, Morgan TC, McBee NA, et al. Minimally invasive surgery plus recombinant tissue-type plasminogen activator for intracerebral hemorrhage evacuation decreases perihematomal edema. Stroke. 2013;44(3):627-34.

12. Nagasaka $T$, Tsugeno $M$, Ikeda H, Okamoto $T$, Takagawa $Y$, Inao $S$, et al. Balanced irrigation-suction technique with a multifunctional suction cannula and its application for intraoperative hemorrhage in endoscopic evacuation of intracerebral hematomas: technical note. Neurosurgery. 2009; 65(4):E826-7 discussion E827.

13. Wang WH, Hung YC, Hsu SP, Lin CF, Chen HH, Shih YH, et al. Endoscopic hematoma evacuation in patients with spontaneous supratentorial intracerebral hemorrhage. J Chin Med Assoc. 2015;78(2):101-7.

14. Nishihara T, Morita A, Teraoka A, Kirino T. Endoscopy-guided removal of spontaneous intracerebral hemorrhage: comparison with computer tomography-guided stereotactic evacuation. Childs Nerv Syst. 2007;23(6):677-83.

15. Dye JA, Dusick JR, Lee DJ, Gonzalez NR, Martin NA. Frontal bur hole through an eyebrow incision for image-guided endoscopic evacuation of spontaneous intracerebral hemorrhage. J Neurosurg. 2012;117(4):767-73.

16. Miller CM, Vespa P, Saver JL, Kidwell CS, Carmichael ST, Alger J, et al. Imageguided endoscopic evacuation of spontaneous intracerebral hemorrhage. Surg Neurol. 2008;69(5):441-6 discussion 446.

17. Abdu E, Hanley DF, Newell DW. Minimally invasive treatment for intracerebral hemorrhage. Neurosurg Focus. 2012;32(4):E3.

18. Nishihara T, Teraoka A, Morita A, Ueki K, Takai K, Kirino T. A transparent sheath for endoscopic surgery and its application in surgical evacuation of 
spontaneous intracerebral hematomas. Technical note. J Neurosurg. 2000; 92(6):1053-5.

19. Rennert RC, Signorelli JW, Abraham P, Pannell JS, Khalessi AA. Minimally invasive treatment of intracerebral hemorrhage. Expert Rev Neurother. 2015; 15(8):919-33.

20. Hsieh PC, Cho DY, Lee WY, Chen JT. Endoscopic evacuation of putaminal hemorrhage: how to improve the efficiency of hematoma evacuation. Surg Neurol. 2005;64(2):147-53 discussion 153.

21. Zhu H, Wang Z, Shi W. Keyhole endoscopic hematoma evacuation in patients. Turk Neurosurg. 2012;22(3):294-9.

22. Orakcioglu B, Beynon C, Bosel J, Stock C, Unterberg AW. Minimally invasive endoscopic surgery for treatment of spontaneous intracerebral hematomas: a single-center analysis. Neurocrit Care. 2014;21(3):407-16.

23. Ma L, Hou Y, Zhu R, Chen X. Endoscopic evacuation of basal ganglia hematoma: surgical technique, outcome, and learning curve. World Neurosurg. 2017;101:57-68.

24. Tada A, Hisada K, Suzuki T, Kadoya S. Measurement volume of intracranial hematoma by computed tomography (author's transl). No Shinkei Geka. 1981;9(3):251-6.

25. $\mathrm{Hu} C L, L Y L Z C$, et al. Guidelines for normal treatment of intracerebral hemorrhage with minimally invasive paracentesis. Beijing: Peking Union Medical College (PUMC) Press; 2003.

26. Anderson CS, Heeley E, Huang Y, Wang J, Stapf C, Delcourt C, et al. Rapid blood-pressure lowering in patients with acute intracerebral hemorrhage. $N$ Engl J Med. 2013;368(25):2355-65

27. Anderson CS, Huang Y, JG Wang H, Arima B, Neal BP, et al. Intensive blood pressure reduction in acute cerebral haemorrhage trial (INTERACT): a randomised pilot trial. Lancet Neurol. 2008;7(5):391-9.

28. Kuo LT, Chen CM, Li CH, Tsai JC, Chiu HC, Liu LC, et al. Early endoscopeassisted hematoma evacuation in patients with supratentorial intracerebra hemorrhage: case selection, surgical technique, and long-term results. Neurosurg Focus. 2011;30(4):E9.

29. Nagasaka T, Tsugeno M, Ikeda H, Okamoto T, Inao S, Wakabayashi T. Early recovery and better evacuation rate in neuroendoscopic surgery for spontaneous intracerebral hemorrhage using a multifunctional cannula: preliminary study in comparison with craniotomy. J Stroke Cerebrovasc Dis. 2011;20(3):208-13.

30. Chen CC, Chung HC, Liu CL, Lee HC, Cho DY. A newly developed endoscopic sheath for the removal of large putaminal hematomas. J Clin Neurosci. 2009;16(10):1338-41.

31. Du B, Shan A, Zhang Y, Zhong X, Chen D, Cai K. Zolpidem arouses patients in vegetative state after brain injury: quantitative evaluation and indications. Am J Med Sci. 2014;347(3):178-82.

Ready to submit your research? Choose BMC and benefit from:

- fast, convenient online submission

- thorough peer review by experienced researchers in your field

- rapid publication on acceptance

- support for research data, including large and complex data types

- gold Open Access which fosters wider collaboration and increased citations

- maximum visibility for your research: over $100 \mathrm{M}$ website views per year

At $\mathrm{BMC}$, research is always in progress.

Learn more biomedcentral.com/submissions 